\title{
Science and Technology Innovation Promotes the Development of City-Industry Integration in Resource-based Cities: a Logical Framework

\author{
Xiao-Jun WANG ${ }^{1, a}$ and Qiang ZHU ${ }^{1, b}$
}

\author{
${ }^{1}$ The Business School of Hunan University of Humanities, Science and Technology, Hunan Loudi, \\ 417000, China \\ awxj1234555@126.com \& 434403541@qq.com, b zhuqiang168@126.com
}

Keywords: City-industry integration, science and technology innovation, resource-based cities.

\begin{abstract}
Single industrial structure and lagged urban construction have severely restricted the development of city-industry integration and new urbanization in resource-based cities. Promoting the city-industry integration with science and technology innovation has become an important path for resource-based cities to achieve transformation, upgrading and sustainable development. Through technological innovation, Resource-based cities would promote transformation and upgrading of industries to consolidate the industrial foundation of city-industry integration, improve the quality and capacity of city to enhance city functions of city-industry integration, lead the construction of science and technology system to optimize the development environment of city-industry integration, promote city's delicacy management to enhance the level of city-industry integration.
\end{abstract}

\section{Introduction}

The city-industry integration development is a kind of development idea compared to city and industry separation under the background of the transformation and upgrading of China's industrial structure and consumption structure [1]. In the early stages of industrialization and urbanization, The urban development zone construction guided by real estate development has spawned a number of industrial "empty cities" and "sleeping cities", with inadequate matching of urban service facilities, incomplete basic functions, and lagging behind in industrial development [2], It has become an important factor in hindering the development of our country's new urbanization. In this respect, At the Third Plenary Session of the 18th CPC Central Committee, it was clearly stated that promoting urbanization based on human beings and promoting integration of industry and town development, and it was formally proposed that advancing the mix of functions and integration of cities and towns and preventing the hollowing out of new urban areas in the National New Urbanization Plan (2014-2020). The city-industry integration development is not only a realistic demand for China's industrial transformation and upgrading, but also an important path for China to accelerate the development of new urbanization. In the development process, resource-based cities generally face the dual constraints of single industrial structure determined by special resources and extensive development methods. There are problems such as excessive consumption of resources, deterioration of the ecological environment, loss of urban population, and gradual aging of urban facilities, etc. The phenomenon of separation of industry and city is more prominent. Under the macro background of removing production capacity, adjusting structure, and transferring modes, resource-based cities are faced with enormous pressures of industrial transformation and upgrading and urbanization construction, and it has become a rational choice for transformation and upgrading of resource-based cities and scientific development through that promoting the integration of industry and cities with science and technology innovation.

\section{Literature Review}

At present, the theoretical research on the development of city-industry integration in China mainly includes the connotation (Zhang Dao-gang, 2011; Li Wen-bin, Chen Hao, 2012). Evaluation System (Su Lin, Guo Bing, Li Xue, 2013; Wang Fei, 2014; Zhang Jian-qing and Shen Yu-wen, 2017), Internal Mechanisms and Development Paths (Li Guang-hui, 2014; Xie Cheng-yang, Hu Han-hui, 
and Zhou Hai-bo, 2016; Yan Bing-feng, 2017), the regional practices (Gao Gang-biao, 2011; Lin Li-jian, Teng Tang-wei, In 2014, Li Yu-xin and Zhang Wei-zheng (2018) and other aspects. They formed a certain degree of consensus on the importance of city-industry integration for the construction of new urbanization. In general, the theoretical research on the integration of city and industry is less and fragmented, and a complete theoretical system has not yet been formed, the research on the integration and development of regional and urbanized cities and towns, especially the research on the integration of city and industry in resource-based cities, is relatively rare. The research on the integration of the production and urban development of resource-based cities with scientific and technological innovation has only been scattered in a small number of policy proposals, and there is still a lack of specialized research. Therefore, this article intends to analyze the characteristics of resource-based cities' city-industry integration, construct a general framework for promoting the integration of city-industry integration in the development of resource-based cities, and explore the strategies for promoting the integration of city-industry integration in resource-based cities.

\section{Main Problems of the City-industry Integration in Resource-based Cities \\ Overexploitation of Resources and Inaccurate Positioning of City-industry Integration Strategy.}

Resource-based cities are the types of cities whose mining and forestry natural resources are exploited and processed as the leading industries in this region. The strategic orientation of the city-industry integration development is determined mainly on the basis of resource endowments, resource development and processing industry, and the spatial layout of resources, has obvious resource-driven properties. Especially in the traditional development model and basic framework of "fighting by mountains, relying on water and draught", it is difficult to break through the inherent resources and industrial advantages to open up innovative industries and related supporting industries, and form an extended industrial chain and industrial clusters. In response to this, most of the limited decision-making processes of government and enterprises are concise or simple, or because of performance-based incentives, the orientation of the city-industry integration is often subject to and serves existing economic foundations and industrial advantages. There is an emphasis on space expansion and neglect of industrial integration, an emphasis on population size and neglects economic support, focuses on development speed and neglects development quality, attaches importance to external policy incentives and neglects endogenous development needs, attaches importance to conditional construction and neglects operation management and other value orientations and development positioning biases. It is difficult to develop intrinsic laws and characteristics of regional economy from the perspective of urbanization. Urban and rural residents' intrinsic needs and other perspectives are well positioned for urbanization development and strategic top-level design, so as to form a "tunnel short-sightedness" in the integration of city and industry, and it is difficult to truly lead the urbanization process and the city-industry integration development.

\section{Single Industrial Structure and Insufficient Industry Support for City-industry Integration.}

Resource-based cities are created due to the development of resources. Resource endowments are both the genes for urban development and the basis for industrial development. Most resource-based urban industrial structures rely mainly on the development and utilization of resources, and are "one industry only." Data shows that the added value of mineral resources development in 262 resource-based cities in China accounts for about $25 \%$ of the total industrial added value, which is more than double the national average, and the proportion of its tertiary industry is $12 \%$ lower than the national average [3]. Such an industrial structure makes the development of resource-based cities vulnerable to regional resource endowments. The abundance of regional resources determines the scale and service life of the resources development industry [4], and even determines the speed, scale, and quality of urbanization. At present, most resource-based cities in China are experiencing economic stagnation and slowing down of urbanization due to resource depletion. At the same time, 
reliance on the lucrative profits brought about by the early and mid-term development of resources, the industrial structure of resource-based cities is generally based on resource extraction and primary processing. There are drawbacks such as insufficient industry deepening, short industrial chain, insufficient industrial clusters, and strong industrial division of labor, which has obviously extrusion effect on non-resource industries. Resource-based industrial structure leads to the lag of development of other connected alternative industries, and most existing resource-based cities fall into the "resource curse" trap because of their excessive resource path dependence.

\section{Incomplete City Functions and Insufficient Support Services for City-industry Integration.}

Resource endowments and geographical distribution determine that resource-based cities are usually distributed in inland areas, remote mountainous regions, or desert areas, with a decentralized spatial layout, poor locational traffic conditions, weak ability to collect factors, and generally insufficient radiation capacity in developed regions and cities. , Cities share weak development capabilities. At the same time, resource-based cities are usually built as resource bases and processing production bases in the early stage of construction, the supply of urban industry development factors and public service facilities, such as culture and education, medical and health care, trade and circulation, leisure and entertainment, and postal communications, human resources intelligence, management consulting, and technological innovation, etc. Is obviously insufficient, and the function of urban comprehensive services is not perfect. In addition, the traditional "three highs and one low" extensive resource extraction and processing methods have caused serious environmental pollution and ecological damage, and resource-based cities are facing more and more serious constraints on resources and the environment. The constantly shrinking resource economic development space and the deteriorating ecological environment have reduced the industrial and population carrying capacity of resource-based cities, aggravated the urban industrial transfer and the outflow of population, and has directly affected the development of the city-industry integration in resource-based cities.

\section{Lack of City Management Concepts and Low Management of City-industry Integration.}

To accelerate the development of city-industry integration in resource-based cities, it requires not only hardware supports such as industrial base and urban facilities, but also software supports such as development strategy and operation management. However, for a long time, due to the characteristics of regional space, urban layout, industry base, and employment threshold, resource-based cities have a value orientation and policy logic to focus on infrastructure construction and ignore urban operation management, and this has led directly to low city management capabilities and level, the degree of city-industry integration and factor management are relatively low. This kind of value orientation and policy logic have been formed because, on the one hand, the spatial layout of resource-based cities is mostly remote and backward, the size of cities is not large, the attractiveness of high-quality resources is not strong, and the difficulty of city management is relatively small; on the other hand, the characteristics of industries dominated by resource extraction and rough processing determine the low employment threshold, single employment structure and relatively low quality of the population, urban population management is relatively easy. At the same time, due to the simple industrial structure and obvious resource characteristics, the source of urban economic development is simple and easy, induce the complex management thinking model and policy value orientation of the city-industry integration to be simplified.

\section{Science and Technology Innovation is a Good Way for City-industry Integration of Resource-based Cities}

\section{Science and Technology Innovation Promotes the Development Power of Resource-based Cities' Industries.}

Under the new normal of economic development, resource-based cities must fundamentally change the mode of development, adjust and optimize the industrial structure, and shift from resource-dependent to innovation-driven. The implementation 0pinions on supporting the industrial 
transformation and upgrading of old industrial cities and resource-based cities, issued by the national development and reform commission and other 5 departments, clearly stated that innovation-driven industrial transformation and upgrading of endogenous motive mechanisms should be established. Therefore, resource-based cities should drive industrial transformation and upgrading through science and technology innovation, and continuously improve the supporting role of the industry in cities ${ }^{[5]}$, Such as research and introduce science and technology innovation support policies, support enterprises to make full use of high and new technology and advanced and applicable technologies to optimize the development of resources deep processing industry, cultivate and expand the succession of alternative industries, improve the utilization of resources through the improvement of technology and management, through the development of resources and local resources relevant circular economy promotes the development of environmental protection and low-carbon industries. By combining its own advantages in resources and geographical features, it will extend the both sides of resource-based industries to promote the high-end and diversified development of the industrial structure and enhance industrial competitiveness.

\section{Promote the Comprehensive Function of Resource-based City by Science and Technology Innovation.}

Science and technology innovation plays an important role in the promotion of town functions, space conservation and utilization, and the development of featured towns. It is also the core driving force for the integration of production-oriented cities in resource-based cities. Resource-based cities should fully rely on scientific and technological innovation and intellectual resources to coordinate production factors such as industry, population, and land, scientifically plan the top-level design of integration of city and industry, launched a series of engineering construction and research projects such as urban function promotion and space conservation and utilization, green energy saving and green buildings, characteristic towns and special industries, urban ecological living environment quality assurance. Through science and technology innovation and intellectual resources, enhance the ability of independent innovation, and provide practical demonstrations for the use of advanced technology to solve the problem of city-industry integration and urban development.

\section{Optimize the Development Environment of the City-industry Integration in resource-based cities with scientific and technological innovation.}

Although various resource-based cities, such as resource endowments, location conditions, and industrial infrastructure, have different economic development performances, they all face low industrial levels, unsound urban functions, and support and protection of industrial upgrading and urban functions in the process of transformation and development. The lack of similar high-level production factors such as technology and talents needed to improve the system is similar ${ }^{[6]}$. Therefore, resource-based cities should innovate its institutional mechanisms, increase the introduction and training of high-tech talents, strengthen the incubation of science and technology innovation teams and the construction of platforms, improve mechanisms for investment in scientific and technological innovation, technological achievements, etc. Promote the economic growth model change from mainly depending on increasing the consumption of material resources to relying mainly on scientific and technological progress and improving the quality of laborers. and promote the city-industry integration in resources-based cities to achieve coordinated and sustainable economic, social and ecological development.

\section{Improve the Management Ability of Resource-based Cities with Scientific and Technological Innovation.}

The city-industry integration in resource-based cities requires not only the improvement of industrial development capabilities and levels, but also the increasing in support and bonding between industrial development and urban management. Accelerating the development of integration of city and industry requires the development of a high-caliber urban management team with sufficient numbers and reasonable structure. It is necessary to optimize urban management and management methods based on the development of modern management technologies, innovate urban 
management methods, and improve the overall capacity of urban management, promote the deep integration of modern industry and city operations. It's all required for the construction of a high-level urban management talent team and the application of modern management technology innovation to establishing a guarantee and support system for scientific and technological innovation. At the same time, scientific and technological innovation can also improve the quality of citizens in an all-round way and enhance the initiative and creativity of the general public in promoting the development of the integration of cities and cities.

\section{Conclusions}

Under the new normal economy, the transformation and development of resource-based cities is a major strategic issue that the country attaches great importance to. The city-industry integration with the help of new urbanization construction is an important approach for the transformation and development of resource-based cities. Scientific and technological innovation will inject new kinetic energy resources into the city-industry integration in resource-based cities. Science and technology innovation can promote industrial transformation and upgrading to consolidate the industrial foundation of the city-industry integration, serve city's quality and renovation improvement to improve urban function of city-industry integration, lead the construction of scientific and technological innovation system to optimize the city-industry integration and urban development environment, promote the city's delicacy management to enhance the city-industry integration management.

Of course, the city-industry integration in resource-based cities involves industries, cities, transportation, population, land, science and technology, environmental protection, management, and systems, etc. This article only focuses on industrial cities from the perspective of science and technology innovation to promote industrial transformation and upgrading and urban function upgrading. The analysis of integration issues provides a viable analytical idea for the city-industry integration of resource-oriented cities, and it is yet to be further explored how resource-based cities can achieve the city-industry integration.

\section{Acknowledgement}

This research was financially supported by Hunan Provincial Social Science Association Foundation: research on optimizing the allocation of financial resources to the real economy in Hunan (No. XSPYBZZ029); Key Project of Education Department of Hunan Provincial: research on the optimization and optimization of financial resources in the process of agricultural modernization in Hunan province (No. 17A112); Hunan Province Social Science Foundation: research on optimized allocation of financial resources in Hunan Wuling mountain area from the perspective of precise poverty alleviation (No. 16YBX010); Loudi Science and Technology Project (No. 3331503).

\section{References}

[1]Li Wen-bin, Chen Hao. Analysis of the Connotation of Production-city Integration and Suggestions for Planning. Urban Planning Journal, S1 (2012): 99-103.

[2] Chen Liu-qin. The integration of city and city to support the development of new urbanization. www.chinareform.org.cn, 2015-07-14.

[3] Du Ying: "Resource-based industries in resource-based cities" issue of "one industry only". http://www.chinanews.com/gn/2013/12-03/5574036.shtml.

[4] Ding Xiang-cheng, Zhang Ying. Research on Economic Transformation of Resource-based Cities Based on Life Cycle Theory. World Geography Research. 3 (2008): 70-76. 
[5]Li Yu-xin, Zhang Wei-zhen. Research on the Development Level of Production-city Integration in the Perspective of New Urbanization: A Case Study of Five Provinces in Northwest China. Research in Business Economy, 1 (2018): 147-149.

[6] Tang Xiao-yu. Thinking of promoting the transformation and development of resource-based cities in our province. Sichuan Daily, 2014-10-09. 